it is a condition of traumatic retino-choroiditis characterized by diffuse cloudiness of the retina, numerous small exudates in the choroid, and fine dust-like opacities of the vitreous. It is identical with retino-choroiditis of secondary syphilis. It varies in degree from a very slight opacity of the vitreous to diffuse thick opacities with changes in the choroid. I have seen a large number of such cases in which I am positive the changes were due to trauma (windage).

Surgery forms one of the most interesting parts of military ophthalmology, though no occasion arises for performing many of the operations of civilian practice. One reason, and one reason alone, should form the basis for all military operative work-necessity. It is most unwise to correct strabismus, for instance, for cosmetic reasons, or to do any operations of a similar nature. If a recruit is accepted for service with a disability of this kind, and an attempt is made to correct it, he first becomes a hospital patient without being ill or wounded (in itself most unlesirable), and secondly he is given the starting point for in attempt to obtain a pension. Dacryocystitis is not nfrequently met with in military work, and for military purposes, and the same would hold among industrial workers in civilian life. I can very strongly recommend the West operation.

Esser, in his article in the Annals of Surgery (March, 1917), forecasts considerable increase in the types in which his method will be useful, and already important progress has been made. Major C. W. Waldron, C.A.M.C., first undertook charge of the face injuries service at West Cliff Canadian Eye and Ear Hospital. This service, in need of greater space, was later moved to a Canadian General Hospital, and later to the Queen's Hospital, Sidcup Major H. D. Gillies, R.A.M.C., and Major Waldron have improved on Esser's methods, and given us a most valuable means of remedying many of the war distortions of the lids by a method of epidermic outlay as compared with Esser's epidermic inlay. Major Waldron has modified Esser's method by making the incision in the conjunctival sac instead of through the eyelid skin. Major Gillies has made two modifications of the Esser process. The first method consists in burying the graft covered mould in the subcutaneous tissues of the eyelid through an incision in the skin, and removed through that incision. By this method the eyelid skin is increased to the extent of the graft. This is especially useful in contractions following burns. The second method may be termed an epithelial overlay.

The subject of military ophthalmology is so large that I have only been able to touch here and there on some of the essential features. I hope, however, that I have been successful in drawing attention to what an essential part of military medicine ophthalmology really is.

It is to the credit of the Canadian Army Medical Service that at a very early period of the war the necessity for a special hospital was noted, and that in October, 1915, the - West Cliff Canadian Eye and Ear Hospital was opened with a capacity of 105 beds. There were 3 administrative and 4 medical officers, 10 nursing sisters, and 27 other iranks. From that beginning the hospital has increased until at the present time it has an establishment of 400 beds, 12 officers, and 40 nursing sisters. There have been admitted to hospital 9,854 cases, and there have been 49,906 consultations in the out-clinic department. Here are prepared the special reports so essential for medical boards, for categorization, for pensions, and for the final disposal of men. Much credit is due to Colonel J. D. Courtenay of Ottawa, who by his initiative had a great deal to do with the establishment of the hospital. By his persistence he saw that it was properly equipped, and by his foresight made the necessary accommodation for its possible growth. The hospital was originally intended to be the head of the Canadian Ophthalmic Service, where civilian ophthalmologists would be sent for military training. Unfortunately, war is a time of changes. We are, however, practising military ophthalmology in as conservative and in as scientific a manner as possible. We have all the equipment, including library and laboratory, necessary for such work, and hope a suficient number of civilian ophthalmologists are being trained for useful military duty. These officers will later be called on to decide many important questions: What eye injuries and diseases are due to service? What is the relation between wounds of the cranium and defective vision? Is loss of central vision without macular changes in soldiers due to service, and is it pathological or functional? What injuries are due to "windage"? What is rational treatment for malingering, functional amblyopia, war neuroses? What is the amount of disability due to haemianopsia? All these and many other questions of a professional nature, and many medico-legal questions, must be decided by us with fairness to the individual and to the State.

\section{A CASE OF FATAL JAUNDICE.} BY

RAWDON A. VEALE, B.A.Oxon., M.D.Lond., M.R.C.P., TEMPORAKY LIEUT.-CoLoNeI R.A.M.C.(T.F.), AND

\section{B. H. WEDD, M.D.LoND.,} CAPTAIN R.A.M.C.

Amongst the complications and sequelae which attend the administration of salvarsan or allied compounds not the least important is that of jaundice. In his paper on the treatment of syphilis Harrison ${ }^{1}$ places jaundice twelfth in his list of clinical side-effects and states that it occurred in 0.6 per cent. of his cases. Deaths due to this cause have been recorded, and consequently we have thought the following case worthy of record.

Pte. P., aged 30, was admitted to a general hospital at 10 p.m. on April 10th, 1918, having been transferred from a casualty clearing station to which he had been admitted on the previous day. His field medical card contained the following entries: "Aching all over for two days. Profuse sweats. Tongue furred. Temperature 1010." The initial diagnosis was influenza. He was obviously gravely ill; his condition, indeed, was such that minute questioning was not feasible. However, he was able to supply the information that he had always been a strong and healthy man, but that he had contracted venereal disease in Italy in the latter part of 1917 and had been subsequently treated witl " injections," details of which are given below. He was distinctly but not deeply jaundiced; he was extremely restless and, though conscious, his mind wandered at times during the examination. Vomiting was frequent and distressing, and the vomitus resembled the black vomit of icterus gravis. The lips were cyanosed and the pulse was weak (100). The temperature $\left(97^{\circ}\right.$ on admission) remained subnormal throughout. There was tenderness over the liver and spleen, but no obvious alteration in the size of these organs. There were no subcutaneous haemorrhages, and no other physical signs worthy of note. The knee-jerks were present. The urine contained albumin, but no other abnormal constituent. Treatment was begun on the lines of free purgation with salines and the administration of alkalis, but the patient grew rapidly worse. He became noisy and wildly delirious, and death tinally occurred on April 12th at 3.30 a.m.

Notes kindly supplied by the medical officer in charge of the patient when under treatment for syplilis provide the following additional information: When admitted to hospital on January 15th, 1918, the man was suffering from a typical hard granulating sore on the corona, which appeared at the end of December, 1917; there was general adenitis, but no other signs or symptoms. He was treated with intravenous injections of 0.6 gram of novarsenobenzol on January 15th, 18th, 21st, February 5th, 8th, 24th, 27th, and March 2nd. No reaction followed on any of these occasions. $\mathrm{He}$ also received intramuscular injections of metallic mercury, as follows: $\frac{3}{4}$ gr. on January 15th, and 1 gr. on each of the following dates: January 22nd, 29th, February 5th, 12th, and March 3rd. There was a sup purating bubo on January 22nd, which was reported healed a week later. On February 24 th a slight stomatitis was noticed. The patient was discharged to duty on March 4th.

Thus it will be seen that there was an interval of some five weeks between the last intravenous injection and the appearance of symptoms of the fatal illness.

Post-mortem Examination.

Body well nourished; definitely jaundiced. Post-mortem staining present. Larynx and trachea normal, as was the oesophagus but for one small submucous haemorrhage. The bronchi sontained blood clot and mucus; there was no fluid in the pleural cavities nor any evidence of pleurisy 
seen. The pleural surface of the lungs showed numerous dark convex areas resembling the condition resulting from infaretion. On section these were found to be due to recent haemorrhages, the majority immediately beneatl the pleura, the rest widely scattered throughout the sub stance of the organ. Portions excised felt partly consoli. dated, but floated in water. The heart weighed $10 \mathrm{oz}$. The muscle was pale; the valves normal. There was no marked alteration in the size of the liver; it weighed $36 \mathrm{oz}$. On section the liver substance was firm, with distinct nutmeg appearance. The gall bladder was normal. The spleen weighed $4 \frac{1}{2}$ oz.; its substance was dark purple moderately firm. The pancreas and suprarenals appeared normal. The kidneys weighed $6 \mathrm{oz}$. each and were some what large and pale. The capsules stripped readily and left a smooth surface. The stomach, duodenum, and intes tines were normal, and nothing abnormal was detected in the brain.

Histological Examination.

We have to express our thanks to Dr. J. A. Murray, of the Imperial Cancer Research Laboratory, who kindly undertook the preparation of these sections, for which we had no facilities, and gave us his opinion upon them.

Liver.-Sections show striking changes. The parenchyma cells are shrunken and separated from one another appearing to have undergone degeneration. There is some overgrowth of the interstitial connective tissue and pro liferation in the portal areas. Tissue for the staining and demonstration of fat was not preserved.

Lung.-The lung shows recent haemorrhages into the alveoli, probably due to infarction. The walls of the alveoli appear normal.

Kidney.-The cells of the tubules show vacuolation probably due to fatty changes. There is little alteration of the interstitial tissue.

1 Quar. Journ. Med., June, 1917.

\section{A CASE OF SUCCESBFUL CAESAREAN SECTION FOR ECLAMPSIA. \\ BY}

E. W. G. MASTERMAN, F.R.C.S., M.D.,

MEDICAL BUPERINTENDENT OF CAMBERWELL INFIRMARY.

IN connexion with the article by Dr. Strachan on toxaemias in pregnancy, which appeared in the BRITISH Medical Journal of August 3rd, and the letter of Dr. McCann on the same subject on August 10th, it may be of interest to report a case of Caesarean section for eclampsia done a few days before the article appeared. I think most medical men will agree that in this case the result has fully justified this method of treatment.

A. R.. aged 31, had enjoyed good health till her marriage. She had first a three months miscarriaye and in 1914 had an eight months child, now living. In 1916, when pregnant for the third time, she had severe albuminuria with anssarca. She refused a bed in Gay's Hospital, but was delivered of an eight months child (which lived four months) at home and afterwards went into the Clapham Maternity Hospital for the kidney trouble. The disease cleared up. In ,1917 she had another miscarriage (cause nnkmown) at three months. On June 3rd, 1918, she was admitted to Camberwell Infirmary, seven months pregnant, with acute bronchitis and sever, seven inunis pregant, wh acte bronchit s and sever cleared up and the blood disappeared from the arine. When she insisted on discharging herself - against strong advice-on she insisted on discharging hersell-against strong advice

July 4 th, there was only a trace of albumin in the urine.
We saw no more of her until July 27 th, when she was brought to the infirmary by ambulance in an acutely delirious condition with the history that she had had during the previous twentyfour hours twenty fits of eclampsia, as well as severe vomiting Within four hours of admission she had four more typical eclamptic fits. The urine proved to be loaded with albumin there was anasarca of the legs, and the patient's condition appeared to be urgent in the extreme. The temperature was $97^{\circ}$. Labour was not due for another month; the cervix felt hard, and the os only admitted two finger-tips. There seemed to be no labour pains. A careful examination failed to reveal any fetal heart sounds, and I concluded that the child was dead.

I decided that ber history and condition justified immediate Caesarean section, and at 10.30 p.m. I performed the operation in the usual way, which I have found advantageous for contruling the haemorrhage-by drawing the uterus outside the abdominal cavity before emptying it. On opening the uterus the clild was found to be dead, with the head lying deeply in the pelvis, necessitating some little force in extrac. tion. The placenta and membranes were removed, and the nterus was sewn up with one layer of deeply placed silkwormgut sutures and an onter layer of spperficial catgut sptures to cover the acar. over the scar. As the patient was clearly unft for further pregnancies, I excised $1 \mathrm{in}$. of the inner pari of each Fallopian tube and bound the proximal stumps in the uterine substance, wering the place over with peritoneal flaps. The abdominal wall was closed in layers in the usual way. The patient lost a very moderate amount of blood, and the whole operation took ust over half an hour.

The result has been very satisfactory. The patient, who was comatose before the operation, was bright and intelligent the next morning. She knew nothing of what had happened, but was delighted to hear what had been done. The temperature reached $100.4^{\circ}$ that day, owing, I think, to a certain degree of bronchitis, but never reached $100^{\circ}$ afterwards. Since the operation there have been no more fits, no vomiting, and no headache. The bronchitis has cleared up, and the urine, which has increased in quantity, now has only a trace of atbumin. The wound has healed, and the stitches have been.removed. The oedema of the legs has quite gone.

The patient is now getting up, and is apparently in normal health (August 30th, 1918).

This brief outline of a case done so recently with such immediately successful results is sufficient, I think, to commend the performance of this straightforward and by no means difficult operation in suitable cases Had I had reason to believe the child was alive at the time of the patient's admission, the argument in favour of Caesarean section would have been still stronger.

\section{'THE TREATMENT OF IRRITAN'T GAS POISONING.}

By J. M. LaZENBY, Captain R.A.M.C.

In the following notes the treatment suggested applies to all cases of irritant gas poisoning when the symptoms are those of acute catarrh of the mucous membranes of the eyes and of the respiratory tract. With varions modifications it has been employed on this ship for the past three months, and gives very satisfactory results. My experience is that success depends entirely on the care with which the method is carried out and the frequency of the treatment.

\section{INSTRUCTTONS TO ORDERLIES.}

1. Before embarkation begins furnish a dressing table with a throat spray, eye bath; Carrel syringe, vaseline, piain gauze cut to size, cotton-wool, jaconet, bandages, and one pint of a warm solution of sodium bicarbonate-10 grains to the ounce. Cover with a clean towel.

2. After embarkation is complete, select all the severe eye cases whose eyelids are closed through photophobia or dried secretion, and place over the eyes a compress of gauze wet with the solution. When the milder cases have been dealt with the severe ones will be ready for treatment, the com presses having unsealed the eyelids and relieved the acute photophobia.

3. Hathe the margin of the lids till all the crusts can be wiped awray with a wet cotton-wool mop.

4. Fill the syringe with solution, and, taking a piece of cotton. wool in the left hand, draw down with it the lower lid. Instil a few drops into the eye from the syringe, and close the lids. Repeat this till all secretion has been washed away-about four times. Dry the skin with cotton-wool.

5. In mild cases leave the eyes uncovered except by the eye shade. In serere cases put on another compress, cover with jaconet, and bandage lightly on.

6. In all cases it is better to smear a little vaseline on the skin to prevent irritation from the discharge.

For the throat (respiratory) cases proceed as follows

1. Fill the spray half full with the solution.

2. The patient sits up and gargles his throat and month with the solution. He then opens his mouth wide and breathes $i$ and out. The spray nozzle is held an inch from the mouth and the jet directed to the back of the throat. The patient must sit up and respire during spraying.

3. Cease when the patient wants to spit out, and repeat four times.

4. When patients are numerous, all cases who can see to use the apparatus should be instructed to carry out the treatment for themselves, using an eye bath for the eyes instead of the syringe.

5. Since suocess depends largely on frequenoy of treatment, patients must be dealt with every three hours. The last appli. patients must be dealt with every three hours. The last applisevere eye cases the compress must be placed in position and secured with a bandage 